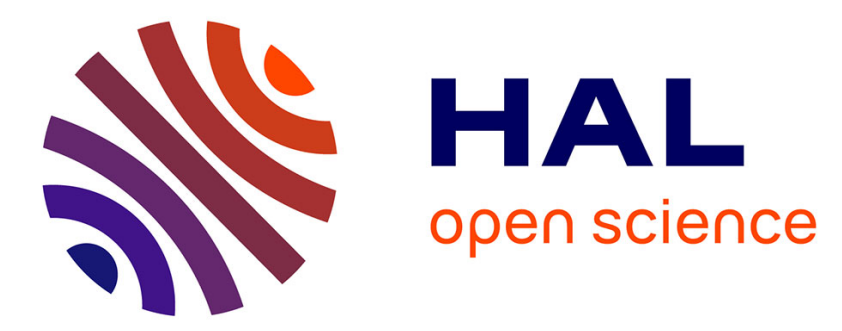

\title{
Comptes rendus des exposés présentés au 2e Colloque d'Aussois sur les Matériaux Supraconducteurs et les Applications de la Supraconductivité Aussois, 24-27 mars 1971
}

Société Française de Physique, Société Française de Métallurgie

\section{To cite this version:}

Société Française de Physique, Société Française de Métallurgie. Comptes rendus des exposés présentés au 2e Colloque d'Aussois sur les Matériaux Supraconducteurs et les Applications de la Supraconductivité Aussois, 24-27 mars 1971. Revue de Physique Appliquée, 1971, 6 (4), pp.481-482. 10.1051/rphysap:0197100604048100 . jpa-00243574

\section{HAL Id: jpa-00243574 https://hal.science/jpa-00243574}

Submitted on 1 Jan 1971

HAL is a multi-disciplinary open access archive for the deposit and dissemination of scientific research documents, whether they are published or not. The documents may come from teaching and research institutions in France or abroad, or from public or private research centers.
L'archive ouverte pluridisciplinaire HAL, est destinée au dépôt et à la diffusion de documents scientifiques de niveau recherche, publiés ou non, émanant des établissements d'enseignement et de recherche français ou étrangers, des laboratoires publics ou privés. 


\title{
COMPTES RENDUS DES EXPOSÉS
}

\author{
présentés au
}

\author{
$2^{\mathrm{e}}$ Colloque d'Aussois sur les Matériaux Supraconducteurs \\ et les Applications de la Supraconductivité
}

\begin{abstract}
AUSSOIS, 24-27 mars 1971
Patronné par

la Société Française de Physique

et la Société Française de Métallurgie avec l'Appui de la D. G.R.S.T.
\end{abstract}

\begin{abstract}
AVANT-PROPOS
Après la tenue en 1968 d'un premier Colloque National sur la supraconductivité, le besoin s'est fait sentir d'une nouvelle mise à jour du sujet, et en particulier, sur les problèmes de matériaux et d'applications, d'où l'orientation assez spécifique donnée à ce Colloque, tenu à Aussois en mars 1971.

Le Comité d'Organisation tient à exprimer ses remerciements à la Société Française de Physique et à la Société Française de Métallurgie pour le soutien qu'elles ont bien voulu manifester, et à la D. G. R. S. T. pour son intérêt et son aide financière, ainsi qu'à tous les participants pour leurs contributions scientifiques et techniques.

Une proportion importante du temps ayant été consacrée à des tables rondes, nous ne publions que les articles de revue, ou les communications, et nous allons tenter de dégager ici les points essentiels discutés par ailleurs.

D'une façon générale, en ce qui concerne les hautes puissances, sauf pour quelques cas particulièrement simples, comme les redresseurs, il est apparu que les études de type technologique sont rarement assez avancées pour que l'utilisateur potentiel puisse chiffrer les caractéristiques minimales pour un développement concurrentiel des techniques basées sur la supraconductivité.

Pour les techniques toujours évolutives, mais maintenant bien éprouvées, liées à la réalisation de bobines à champ continu, des échanges fructueux ont eu lieu en particulier en ce qui concerne la stabilisation minimale, la protection des grosses bobines, la distinction toujours délicate entre saut de flux localisé et début de transition. Un nouveau chapitre s'ouvre avec les études des bobines à champ rapidement variable nécessitées par les accélérateurs, et en particulier au C.E. R. N. Un pas nouveau est accompli par la réalisation et l'emploi de composites multifilamentaires torsadés. Bien sûr, il ne s'agit pas là de fréquences industrielles, mais la réalisation permettra de tester la validité des prévisions et l'on peut penser qu'alors, une extrapolation pas trop hardie, donnera la possibilité de chiffrer beaucoup plus sérieusement les pertes et le comportement à $50 \mathrm{~Hz}$.

Sur le plan de la métallurgie, pas de nouveautés sensationnelles, comme il fallait d'ailleurs s'y attendre puisqu'aucun supraconducteur révolutionnaire n'est apparu depuis 3 ans. Il s'agit ici de travail en profondeur axé plus particulièrement sur les techniques de couches minces, des diagrammes d'équilibre, le rôle joué par l'écrouissage et les revenus dans les courants critiques. Les problèmes de gainage, d'états d'interfaces ont été évoqués, rendus plus actuels par l'utilisa-
\end{abstract}


tion des câbles multifilamentaires. Les composés type $(\mathrm{NbN})$, ou A $15\left(\mathrm{Nb}_{3} \mathrm{Sn}\right)$ sont encore dans une période évolutive. Ainsi l'utilisation de la sonde de Castaing a pu mettre en évidence que la couche de $\mathrm{Nb}_{3} \mathrm{Sn}$ dans la méthode par diffusion est inférieure ou égale à $1 \mu$ alors que par métallographie on évaluait sa largeur de 3 à $7 \mu$.

La demi-journée consacrée aux courants faibles a montré le grand intérêt manifesté pour l'effet Josephson, dans ses applications à la métrologie fine, qu'il s'agisse de magnétométrie, de mesures de tensions, ou même de spectrométrie millimétrique appliquée à l'Astronomie.

Sur le plan des problèmes scientifiques, liés aux applications, un grand intérêt est toujours porté à l'origine, la mesure, et l'efficacité des forces d'ancrage des vortex. La nécessité d'expériences où la distribution et la nature de ces forces sont bien connues, ressentie lors du premier colloque, a fait naître de belles mesures sur des alliages $\mathrm{Pb}-\mathrm{Bi}$ à concentration modulée avec la position suivant une loi imposée. Si dans de nombreux cas, la loi de force entre un vortex isolé et un défaut donné peut être chiffrée théoriquement, le problème de l'efficacité statistique ne semble pas résolu.
Les phénomènes transitoires faisant l'objet d'un article de revue, nous ne retiendrons que la possibilité théorique d'obtenir des supraconducteurs réellement autostabilisés par augmentation de $I_{\mathrm{c}}(T)$ avec $T$. Malheureusement, les supraconducteurs industriels semblent mal se prêter à la réalisation d'inclusions normales de dimensions voulues susceptibles de provoquer un tel effet.

Les échanges thermiques ont également suscité un vif intérêt étant donné leur importance dans toute application, y compris en courant continu pour les problèmes de stabilisation.

En conclusion, il apparaît que le problème est encore très évolutif. Sur le plan technique, l'apparition des multifilamentaires, les projets d'utilisation avec des $\mathrm{d} H / \mathrm{d} t$ élevés, permettent difficilement de rejeter sans appel, des projets du genre alternateur synchrone à excitateur supraconducteur.

Sur le plan scientifique on peut dire que nous ne possédons pas encore de théorie suffisamment fondée des problèmes de force d'ancrage. Les matériaux composites peuvent également donner des résultats surprenants comme les verres poreux imbibés de Plomb ! 